\title{
DÜBLIN
}

Technological University Dublin

ARROW@TU Dublin

\section{Targeting Lipoprotein Biogenesis: Considerations towards Antimicrobials}

\author{
Toufic El Arnaout \\ Technological University Dublin, toufic.elarnaout@tudublin.ie \\ Tewfic Soulimane \\ University of Limerick
}

Follow this and additional works at: https://arrow.tudublin.ie/schfsehart

Part of the Lipids Commons, and the Other Chemicals and Drugs Commons

\section{Recommended Citation}

Toufic El Arnaout, Tewfik Soulimane, Targeting Lipoprotein Biogenesis: Considerations towards Antimicrobials, Trends in Biochemical Sciences, Volume 44, Issue 8, 2019, Pages 701-715, ISSN 0968-0004, DOI: 10.1016/j.tibs.2019.03.007.

This Article is brought to you for free and open access by the School of Food Science and Environmental Health at ARROW@TU Dublin. It has been accepted for inclusion in Articles by an authorized administrator of ARROW@TU Dublin. For more information, please contact arrow.admin@tudublin.ie, aisling.coyne@tudublin.ie, gerard.connolly@tudublin.ie.






\title{
Targeting Lipoprotein Biogenesis: Considerations towards Antimicrobials
}

\author{
Toufic El Arnaout ${ }^{1,2, \star}$ and Tewfik Soulimane ${ }^{3,4}$
}

Decades have passed without approval of a new antibiotic class. Several companies have recently halted related discovery efforts because of multiple obstacles. One promising route under research is to target the lipoprotein maturation pathway in light of major recent findings and the virulence roles of lipoproteins. To support the future design of selective drugs, considerations and priority-setting are established for the main lipoprotein processing enzymes (Lgt, LspA, and Lnt) based on microbiology, biochemistry, structural biology, chemical design, and pharmacology. Although not all bacterial species will be similarly impacted by drug candidates, several advantages make LspA a top target to pursue in the development of novel antibiotics effective against bacteria that are resistant to existing drugs.

\section{Antibiotic Design by Targeting Lipoprotein Maturation and Multi-Objective Planning}

Bacteria versus Hydrophobic Antimicrobial Designs: An Unpredictable Contest

Despite the importance of antibiotic development, leading pharmaceutical companies have recently halted related projects owing to several challenges (Box 1). However, new antibiotics, particularly from novel classes, will be necessary to tackle the emerging crisis of bacterial antimicrobial/antibiotic resistance.

Potential druggable sites may be predicted using bioinformatics tools and protein structures. In the case of the three main enzymes, the topic of this review (Figure S1 in the supplemental information online), these sites are generally accessible from the membrane bilayer and are located at the periplasmic side of the cytoplasmic membrane. It might be easier for drugs to reach these sites than having to cross the cytoplasmic membrane entirely. Nonetheless, drugs must cross layers, diffuse in the cytoplasmic membrane, bind, and have hydrophobic properties. The chemical design needed for such drug characteristics is difficult to predict. Molecules can violate the predicted balance of aqueous versus membrane solubilities, as well as many criteria in the 'Golden Triangle' and the Lipinski's 'rule of five' (Ro5) [1,2] which evaluate properties related to stability, absorption, permeability, potency, distribution, metabolism, and clearance (e.g., ADMET criteria, see Glossary). Lipophilicity in drugs can be challenging [3] and can limit drug candidates during prediction and selection steps. Another difficulty may be in adding lipophilic properties to peptidomimetic scaffolds [4], which complicates it further in terms of rigid conformations, robust synthesis protocols, stability, resistance against degradation, and membrane penetration efficiency [5]. Nevertheless, molecules often prematurely considered unattractive for drug development pipelines sometimes pass clinical development and have a distinctive membrane permeability [6]. The differences between bacterial species also have impact on how effective a drug will be, such as the envelope and membrane composition, the survival mechanisms, and the lipoprotein processing enzymes and lipoprotein forms they possess (Figure 1). Overall, the antibiotic discovery pipeline employs disciplines including microbiology, biochemistry, structural biology, pharmacology, organic chemistry, chemical synthesis, and formulation.

\section{Highlights}

The characteristics of the lipoprotein processing enzymes Lgt, LspA, and Lnt imply lipophilic, membrane-penetrating antibiotic designs, of which the pharmacological qualities for administration must be evaluated cautiously.

Each enzyme varies in relevance across bacterial species, some of which also possess multiple genes and/or functional homologs. Other enzymes and lipoprotein forms may also exist.

Structural comparison of Lgt with other families (PgpB and PIsY) indicates unique insights into inhibition and the lipidbinding cleft.

The mechanism of binding of the LspA inhibitor globomycin requires further studies. Several hypotheses are provided on its inhibition type and interactions with the catalytic site and protein in general.

A novel LspA inhibitor, 'inhibitor-99', shares similarity with known binders of various proteins.

Lnt has four catalytic residues, to which substrate access is controlled by a unique lid-loop.

There is an additional pocket in Lnt for binding and stabilization that lies above the catalytic residues: lessons learned from other protein families (DHHC2O and $\mathrm{PISC}$ ).

\footnotetext{
${ }^{1}$ Kappa Crystals Ltd, Dublin, Ireland ${ }^{2} \mathrm{~S}$ chool of Food Science and Environmental Health, Technological University (TU) Dublin City Campus, TU, Dublin, Dublin, Ireland
} 
Box 1. General Challenges in Bringing a New Antibiotic to the Market

It is increasingly difficult for new molecules in general to reach the market, and biopharmaceutical research and development returns dropped from 10.1 to $1.9 \%$ between 2010 and 2018 [86]. In the context of antibiotics, some of the challenges are:

(i) Slow and costly research process to discover a potential antibiotic.

(ii) Very short (small) return on investments.

(iii) Extremely low antibiotic discovery and approval rates compared to other drug types.

(iv) A regulatory burden throughout the process of validating and approving a new antibiotic.

(v) An antibiotic is usually consumed for a short period, and new antibiotics can be expensive. Clinicians will only use them as a last resort, may not prescribe them immediately, to try to prevent the emergence of resistance.

(vi) Drug delivery obstacles owing to the complex chemical properties of antibiotics, the physiological location of bacteria in the host organism, and/or bacterial protection (e.g., membrane, biofilms, $\mathrm{pH}$ modulation).

(vii) Genetic and microbiological differences between species; an antibiotic may be effective against some species but not against others (examples are highlighted here in the context of lipoprotein biogenesis enzymes), and antibiotic resistance mechanisms may be stronger in some species than in others $[84,85]$.
In this review, to support antibiotic development efforts, interpretations related to these disciplines are therefore raised for the three main lipoprotein maturation enzymes, Lgt (diacylglyceryl transferase), LspA (signal peptidase), and Lnt ( $N$-acyltransferase), motivated by recent discoveries such as the elucidation of their crystal structures [7-12]. The substrates of these enzymes are pre-prolipoproteins, prolipoproteins, and apolipoproteins, respectively [13]. Lipoproteins are encoded by $1-3 \%$ of the bacterial genome, and this corresponds to 30-80 genes on average. Lipoproteins have different modified forms (Figure 1) and are involved in diverse functions such as cell division, adhesion, infection, and virulence. Furthermore, we have carried out analyses of enzymes with similarities to the main lipoprotein maturation enzymes, but that are from different families, as well as analyses of several binding molecules and inhibitors, to highlight enzymatic and inhibition mechanisms from unique viewpoints. Finally, we discuss the targets of foremost potential impact for drug targeting among Lgt, LspA, and Lnt.

\section{Species Dissimilarities and Other Pathway Enzymes: Implications for Novel Antibiotics}

A summary of several possible lipoprotein modification pathways (Lgt, different LspAs and Lnts, Lit, deacylase) is shown in Figure 1. Currently, among these enzymes, the standout candidates for drug discovery are Lgt, LspA, and (classic) Lnt because they are well characterized both functionally and structurally [7-12]. Lgt, LspA, and Lnt are not essential or present in all types of bacteria, mostly depending on the bacterial $\mathbf{G C}$ content classification, membrane properties (i.e., Gram-positive, G+; or Gram-negative, G-) and envelope architecture, and the presence of functional homologs [14-21]. Of note, no significant homologs have been identified in Archaea, although the substrate lipobox sequence is conserved [22].

In $\mathrm{G}$ - bacteria, the trio is generally considered to be essential, although recent studies showed that this is not always the case for Lnt, and diacylated lipoproteins (i.e., not modified by Lnt) may also still be secreted to the outer membrane without being triacylated [14,15]. Furthermore, Myxococcus xanthus, a G- bacterium that produces myxovirescin (an LspA inhibitor), has four IspA genes [16].

In $G$ + species the essentiality of Lgt and LspA also varies [17]. In some mycobacteria Lgt may be essential, but LspA is not [17]. Some G + bacteria (e.g., Clostridium difficile) express two nonidentical LspAs (LspA and LspA2) [18,19]. LspA can also act independently of Lgt [18,23]. Streptomyces spp. (some produce globomycin, an LspA inhibitor) encode two Lgt homologs and two Lnt homologs [20]. Furthermore, in G + bacteria, the usual (E. coli-type) Lnt is generally present in
${ }^{3}$ Bernal Institute, University of Limerick, Limerick, V94 T9PX, Ireland ${ }^{4}$ Department of Chemical Sciences, University of Limerick, Limerick, V94 T9PX, Ireland

${ }^{*}$ Correspondence: toufic.arnaout@kappacrystals.com (T. El Arnaout). 


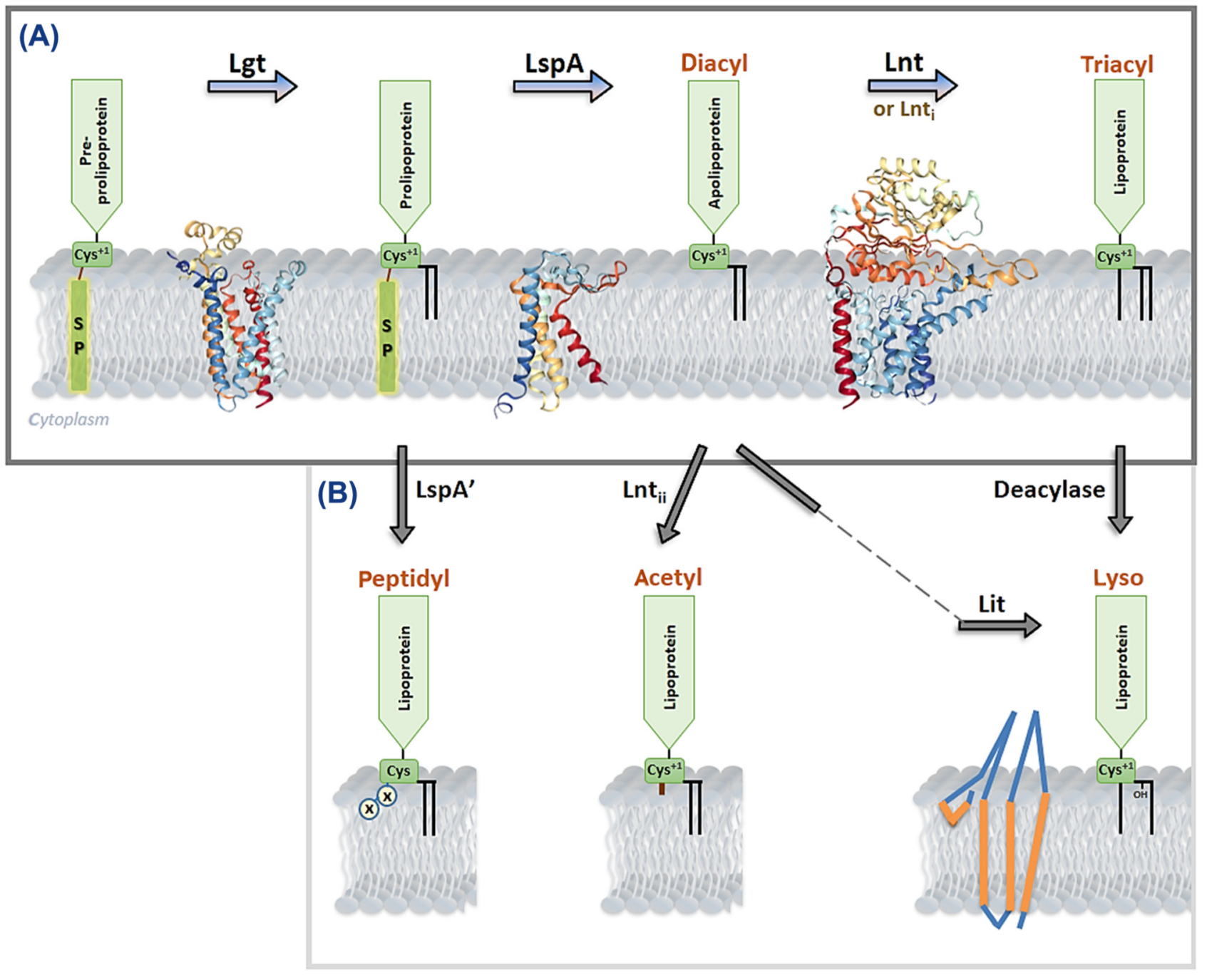

Trends in Biochemical Sciences

Figure 1. An Illustration of Different Lipoprotein Modification Pathways. (A) The classic Lgt/LspA/Lnt pathway [13] showing the membrane proteins Lgt (preprolipoprotein diacylglyceryl transferase), LspA (prolipoprotein signal peptidase), and Lnt (apolipoprotein $N$-acyltransferase). The solved structures of the three enzymes are shown (PDBs 5AZC, 5DIR, and 5N6L [7,8,11]), together with the lipoprotein modifications at each step. Briefly (and not shown), a typical pre-prolipoprotein sequence has an $\mathrm{N}$-terminal signal peptide (SP), followed by a conserved cysteine (the first residue, Cys ${ }^{+1}$, of the mature protein). The pre-prolipoprotein has a 'lipobox motif' with four characteristic amino acids numbered $-3,-2,-1$ and Cys ${ }^{+1}$. Residues -3 to -1 correspond to the last three C-terminal residues of the SP. Lipid modification by Lgt occurs at $\mathrm{Cys}^{+1}$ of the lipobox, then LspA cleaves between residues -1 and Cys ${ }^{+1}$ to remove the SP, and Lnt adds an acyl chain to Cys ${ }^{+1}$. Once processed by all three enzymes, the final lipoprotein is triacylated and lacks the signal peptide. In studied Gram-positive (G+), low GC-content bacteria [21,25], 'Lnt;' 'seemingly has a different sequence and structure from the E. coli-type Lnt. (B) Additional lipoprotein forms (peptidyl, acetyl, and lyso [25]) and their processing enzymes in some G+ bacteria of low GC content. The peptidyl form results from proteolytic processing by an unusual LspA (LspA) two residues before the Cys ${ }^{+1}$. The acetyl form is probably generated by a different enzyme ('Lntii ') rather than by Lnt. The lyso form results from either Lit (intramolecular transacylase [24]) or a deacylase, using the diacyl or triacyl form, respectively. The topology shown for Lit is our predicted model (although a study predicted four rather than three transmembrane helices [24]).

GC-rich species, whereas in low GC-content species a different and unusual Lnt may facilitate triacylation (Lnti) [21]. Interestingly, low GC-content G+ species may harbor additional modification enzymes (e.g., Lit, Lnti, deacylase) and lipoprotein forms that are also unusual [24,25], as described in detail in Figure 1. 


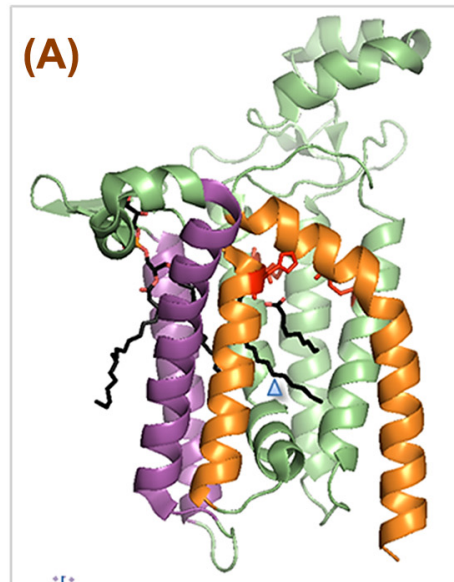

$\frac{1}{190^{\circ}}$

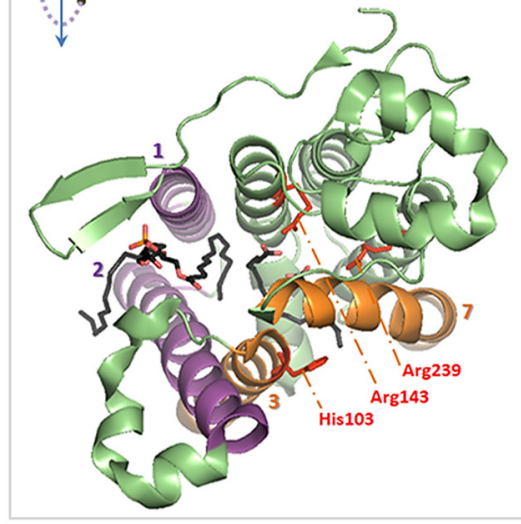

(B)

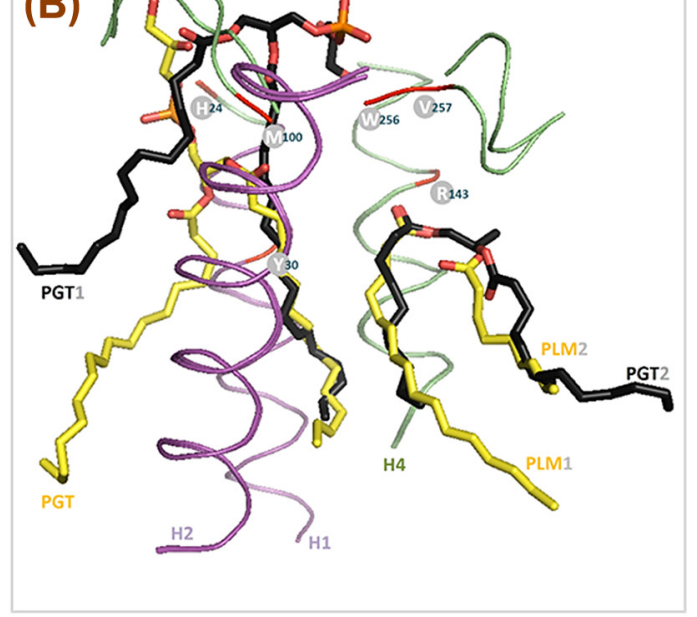



PgpB

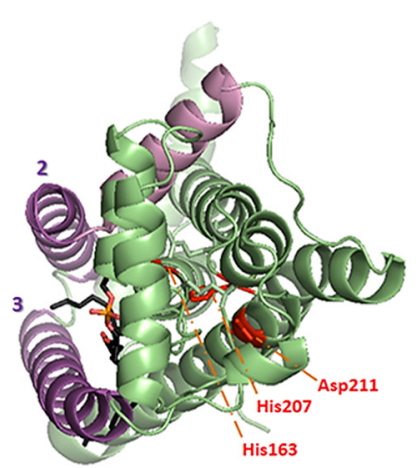

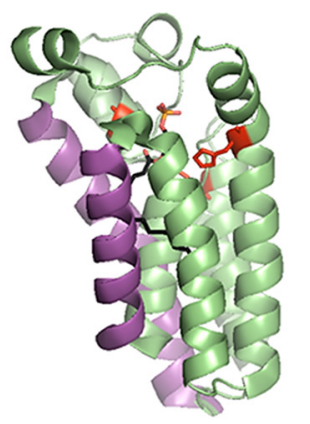

PIsY

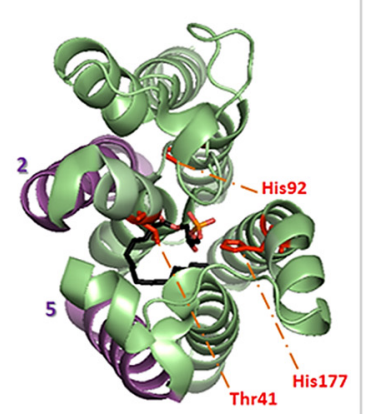

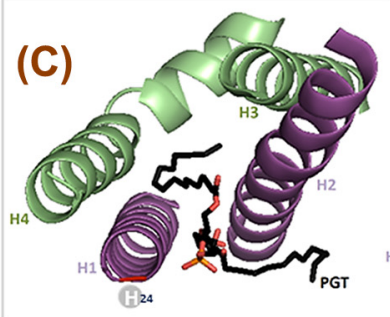

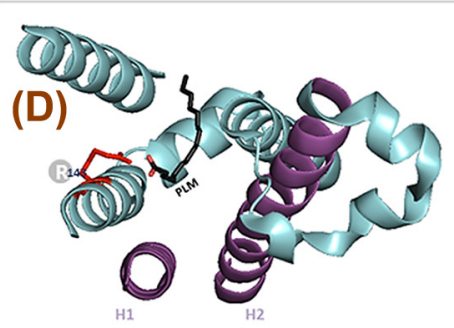

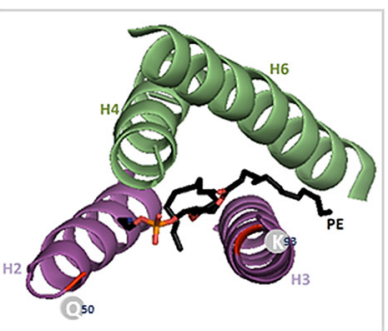

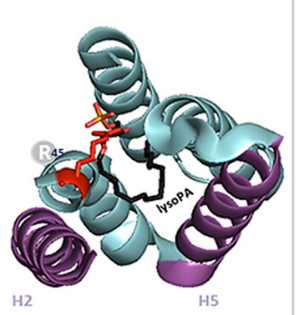

Trends in Biochemical Sciences

Figure 2. The Gating Transmembrane Helices (TMHs) and Lipid Inhibition in Lgt, PgpB, and PIsY. (A) The structures viewed from the front (top row) and from the periplasm (Lgt and PgpB) or cytoplasm (PIsY) (bottom row) (PDBs 5AZB, 5JWY, and 5XJ8 [7,32,33]). Lgt, PgpB, and PlsY are bound to the inhibitors palmitic acid 
Other than the microbiology and genomics described above, we further elaborate on Lgt, LspA, and Lnt in the following three sections to guide additional understanding of each enzyme and the design of selective antibiotics.

\section{Lgt: Binding and Inhibition Insights Based on Phospholipid Biosynthesis Enzymes}

Two Binding Clefts and an Induced-Fit Mechanism Characterize Lgt

Lgt has seven transmembrane helices (TMHs). TMHs 1-2 constitute the 'front cleft' through which binding of the lipid-donor phosphatidylglycerol (PG) occurs in site 1. PG may also slide into an interior site 2, the catalytic site (Figure 2A). Furthermore, TMHs 3 and 7 constitute the 'side cleft' for binding the acceptor domain of the protein [7]. Lgt has a conserved HGGL motif that is important for transacylation (this motif contains His103 in the side cleft) (Figure 2).

In the two solved structures, site 2 was occupied by diacylglycerol (DAG) or palmitic acid (inhibitor); DAG likely originated from PG hydrolysis [7]. In site 1, PG directly interacts with Met100, Trp256, and Val257, or with His24, while in site 2 both DAG or palmitic acid interact with Arg143/Tyr30 [7] (Figure 2A,B and Figure S2). Arg143 and Arg239 are close to each other and are vital for diacylglyceryl transfer (Figure 2A).

Inhibition of the entry and binding of the lipid donor PG at site 1, or at site 2 , is therefore important to restrict the enzymatic activity of Lgt towards the acceptor pre-prolipoprotein. Note that two potential mechanisms [7] follow PG entry into site 1 (initially independent of pre-prolipoprotein binding): (i) PG slides into site 2 (the catalytic site) and the reaction occurs upon binding of the acceptor at site 2, or (ii) PG only slides into site 2 owing to induced-fit and gate control in Lgt that is associated with acceptor binding at site 2. PG sliding may be controlled by a gate close to Arg143. It is important to determine whether the main target should be (i) site 1, (ii) site 2 (from site 1, probably based on an induced-fit structure), or (iii) perhaps the side cleft. The inhibited protein form with palmitic acid may provide insights into specific interactions for drug candidates (Figure 2B), although palmitic acid is not a strong or specific inhibitor. Further, palmitic acid cannot be used clinically because it binds to many proteins [26] and interacts (as in Lgt) with an arginine in kinases, nuclear factors, albumins, cytochromes [27], and a-dioxygenases [28]. Therefore, structurefunction studies of $\mathrm{Lgt}$ with more specific inhibitors will be necessary for future drug design. Specificity must be developed by exploring the unique features of $\mathrm{Lgt}$ versus similar proteins from other families - such as unique interaction residues and lipid-binding pocket properties - or by targeting the side cleft instead of the lipid-binding cleft.

Structural Comparison with Phospholipid Biosynthesis Enzymes for Specific Lgt Inhibitor Design Lgt binds a PG as a donor. Enzymes from other families, particularly those involved in phospholipid synthesis pathways [29], also bind or produce similar lipids during their reactions, and many are membrane proteins with TMHs and a lipid-binding gate as in Lgt [7]. It is remarkable how each may selectively react with, produce, or undergo inhibition by lipids of strong resemblances. Hence, we compare Lgt with two other membrane enzymes, PgpB (phosphatidylglycerol-phosphate phosphatase B) and

\section{Glossary}

ADMET: absorption, distribution, metabolism, excretion, and toxicity. These properties of a potential drug are studied during development to understand the effects on an organism and the suitability and safety for administration.

Amidase: an enzyme from the large family of hydrolases; amidases catalyze the hydrolysis of an amide bond.

Diacylglycerol (DAG): an intermediate formed during some enzymatic reactions involving lipids; also a hydrolysis product of many phospholipids.

ESKAPE pathogens: specific pathogens (Enterococcus faecium, Staphylococcus aureus, Klebsiella pneumoniae, Acinetobacter baumannii, Pseudomonas aeruginosa, and Enterobacter species) that are often cited in the context of antibiotic resistance.

G3P acyltransferase (GPAT): examples of GPAT enzymes in this review include PIsB and PIsY. GC content: guanine and cytosine content (percentage) in genomic DNA. Glycerol-3-phosphate (G3P): used together with an acyl donor by the proteins PIsY or PlsB to produce the phospholipid, Iysophosphatidic acid (IysoPA).

Gram-negative (G-): bacteria such as Escherichia coli and Pseudomonas aeruginosa. They usually have a very thin layer of peptidoglycan and an outer membrane.

Gram-positive $\left(\mathbf{G}_{+}\right)$: these bacteria usually have a thick cell wall with layers of peptidoglycan and no outer membrane. Lipobox: it contains a motif of four residues in the lipoprotein precursor, which are the last three (C-terminal) residues of the $\mathrm{N}$-terminal signal peptide (10-20 residues), and the conserved cysteine that follows (this cysteine becomes the first residue in the mature protein). Lgt adds a diacylglyceryl moiety onto the cysteine, LspA cleaves the

\footnotetext{
(marked with a blue triangle, in site 2, close to Arg, behind phosphatidylglycerol, PG), phosphatidylethanolamine (PE), and lysophosphatidic acid (lysoPA), respectively. The TMHs in magenta (numbered) are likely the gates for lipid binding. In PgpB the upper diverging part related to TMH2 (named the a1-helix [30]) is in lighter magenta. Selected important residues are labeled as red sticks. The TMHs in orange in Lgt are the binding side (side cleft) of the protein substrate. (B-D) Selected regions of Lgt, PgpB, and PIsY. Lipids are shown as sticks. Residues according to the direct interaction maps (Figures S2 and S3) are colored in red and labeled with grey circles. (B) Inhibited (PDB 5AZB, lipids in yellow) versus normal Lgt (5AZC, lipids in black) forms (front view as in A, top), with lipids at site 1 (at TMHs (H) 1 and 2) and site 2 (further behind). Site 2 is where DAG (PGT2) or palmitic acids (PLM2 and the inhibitor PLM1) are bound. Selected interactions are with residues Y, R, and H (5AZB), or Y, R, M, W, and V (5AZC). (C) Top views towards the gate regions of inhibited Lgt (left) versus inhibited PgpB (right) forms, with the bound lipids at the gates (substrate PG in Lgt or inhibitor PE in PgpB). (D) Views towards the gate regions of inhibited Lgt (left) versus inhibited PIsY (right) forms, with the inhibitors palmitic acid and lysoPA, respectively. The figures were generated using the PyMOL Molecular Graphics System (version 1.7.4.5 Edu, Schrödinger, LLC).
} 
PIsY [glycerol-3-phosphate (G3P) acyltransferase], whose structures in complex with lipid inhibitors were recently solved (Figure 2).

PgpB (PDB 4PX7 [30]) appeared as a hit when using an online bioinformatics tool to search for proteins with similar patterns and PDB structures to Lgt [31]. PgpB has prokaryotic and eukaryotic homologs, and its structure with an inhibitor (Figure S3), phosphatidylethanolamine (PE) was also determined (PDB 5JWY [32]). The two enzymes have some structural similarities (Figure 2A, $\mathrm{C})$, but three main residues in $\mathrm{PgpB}$ are more tightly positioned compared to those selected in Lgt (Figure 2A) because Lgt has a more stretched structure. This may be explained by the features of both proteins: PgpB has a front cleft, whereas Lgt has both front and side clefts, as well as visible sites 1 and 2. The side cleft of Lgt (highlighted as TMHs in orange in Figure 2A) has the TMHs positioned more suitably for binding to the pre-prolipoprotein lipobox than TMHs located at a similar angle in PgpB and PlsY (note, unlike Lgt and PgpB, the orientation of PlsY is towards the cytoplasm). Therefore, this cleft may provide a more specific target for designing inhibitors than the front cleft for binding lipid donors.

PIsY is also a membrane protein and a transferase like Lgt. Unlike PgpB, PlsY has no eukaryotic homolog. It catalyzes a reaction (acyl-phosphate + G3P) to form lysophosphatidic acid (lysoPA), thus involving molecules smaller than those processed by Lgt. Interestingly, the structure of PlsY was recently solved in the presence of an inhibitor (the product lysoPA, Figure S3) [33]. PlsY is smaller than both Lgt and PgpB, and has a compact arrangement (Figure 2A). In Lgt, the side cleft TMHs are very close to each other at the top, and then become distant towards the bottom, unlike the corresponding TMHs in PgpB and PIsY. Furthermore, lysoPA is inside the structure of PlsY, but palmitic acid in Lgt is deeper (Figure 2A,D). Both lysoPA and palmitic acid interact with an arginine residue that is crucial for the activities of Lgt and PIsY. Interestingly, His177 in PIsY, that is mainly indispensable for substrate positioning [33], seems at a similar position to His103 in Lgt relative to the lipid-binding gate and the bound lipids inside the structure (site 2 in Lgt).

\section{LspA: Drug Design Recommendations Based on Four Distinct Molecules}

Globomycin: Detailed Hypotheses to Clarify Its Controversial Mechanism

Globomycin $[34,35]$ is an antibiotic lipopeptide (cyclodepsipeptide) which diffuses in the membrane to the protein-binding site of LspA. The sensitivity of $\mathrm{G}$ - bacteria to globomycin may be inversely related to their lipopolysaccharide chain lengths [36]. Modifying the amino acid composition of globomycin can play a role in the antibacterial efficacies of analogs, but in particular a long alkyl side chain (Figure 3 ) is important and its exact length may make globomycin even more effective against $\mathrm{G}$ - bacteria, and to also become effective against $\mathrm{G}+$ bacteria [37]. Such studies are relevant to consider when optimizing species-specific analogs or synthesizing novel peptides based on the essential features of globomycin.

Globomycin was cocrystallized with LspA [8] (Figure 3A). Its Leu-lle-Ser sequence mimics the first three lipobox residues [8] of the prolipoprotein [which can be $(\mathrm{LVI})^{-3}(\mathrm{ASTVI})^{-2}(\mathrm{GAS})^{-1}$ ], in other words the last three $\mathrm{C}$-terminal residues of the signal peptide. The structure of globomycin does not include a mimic of the fourth lipobox residue $\left(\right.$ Cys $\left.^{+1}\right)$ and its linked DAG (Figure 1), nor of residues +2 and +3 in the prolipoprotein substrate that would mimic the binding conformation of substrate above the LspA aspartates (D123 and D143) at the membrane leaflet (Figure 3A). Globomycin may be more of a mimic of the cleaved signal peptide product (it is common for many proteases to be inhibited by product peptides).

In addition, despite binding at active aspartate residues of LspA, globomycin is not a 'competitive' inhibitor as claimed [8] but is 'partially' noncompetitive. In fact, the classification may depend on several molecular and experimental conditions [38], and recently globomycin was suggested to signal peptide at the $\mathrm{N}$-terminus of the cysteine, and Lnt transfers an acyl chain onto the cysteine.

Low-barrier hydrogen bond (LBHB): a short (less than $~ 2.5 \AA$ ) and strong bond with midway hydrogen (certain spectroscopic methods are necessary to confirm this).

Nitrilases: a large superfamily of enzymes, with multiple branches, that hydrolyze non-peptide carbon-nitrogen (CN) bonds; sometimes referred to as CN hydrolases.

Peptidomimetic: a molecule designed to mimic certain properties of a peptide. This has advantages in drug research, for instance in target inhibition. Peptidomimetics are often based on a known peptide but with some modifications.

PgpB: phosphatidylglycerol-phosphate phosphatase B. It plays a role in bacteria membranes in generating phosphatidylglycerol (PG) by dephosphorylating PG-phosphate (PGP).

PIsY (YgiH): a GPAT that produces lysoPA.

POPE: 1-palmitoyl-2-oleoyl-sn-glycero3-phosphoethanolamine (16:0-18:1 phosphatidylethanolamine); a substrate for Lnt.

Steric compression: it is a characteristic of a certain steric effect (sometimes using terms such as crowding or hindrance) and configuration in the binding mode of a molecule (here, an inhibitor). The binding mechanism can initially be slow. Further experiments (e.g., using nuclear magnetic resonance, NMR) are necessary to measure chemical shifts and validate the hypothesis if this applies to globomycin. $\mathbf{X}$-bonding: halogen bonding involving a halogen atom. The classic type otherwise is hydrogen bonding. 


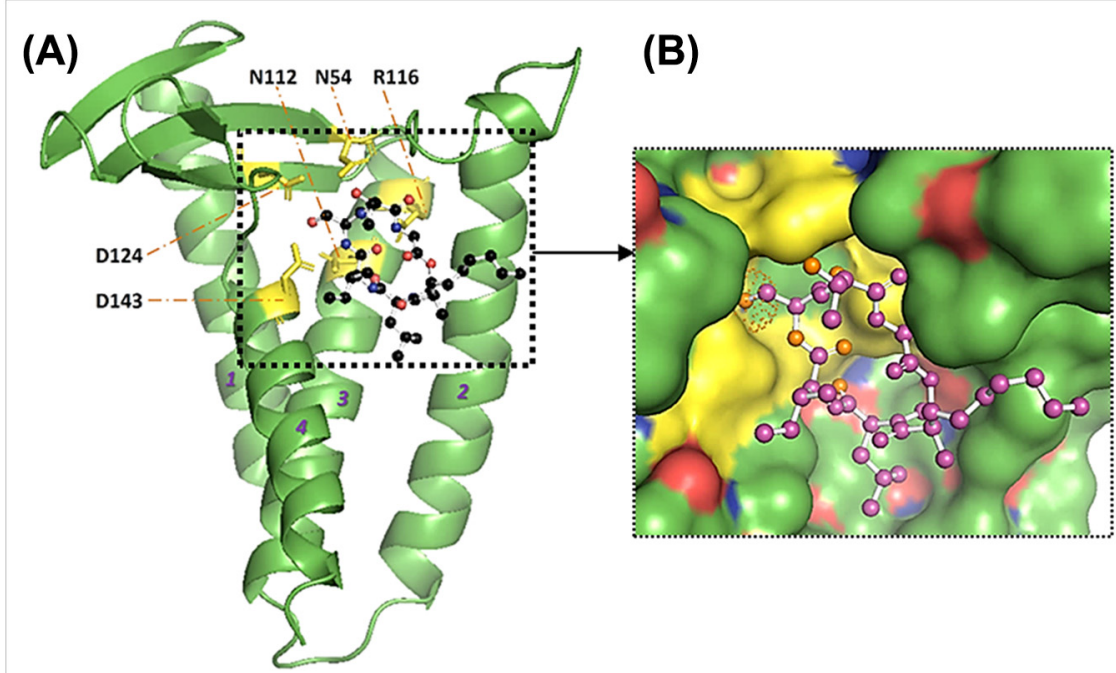

LspA-globomycin
(C)



\begin{tabular}{lcc} 
& $\mathrm{R}$ & $\mathrm{n}$ \\
\hline Globomycin (1a) & $\mathrm{CH}_{3}$ & 3 \\
SF-1902 $\mathrm{A}_{5}$ (1b) & $\mathrm{CH}_{3}$ & 5 \\
SF-1902 $\mathrm{A}_{4 \mathrm{a}}$ & $\mathrm{CH}_{3}$ & 4 \\
SF-1902 & $\mathrm{CH}_{3}$ & 1 \\
SF-1902 A & $\mathrm{H}$ & 3 \\
SF-1902 A & $\mathrm{H}$ & 5
\end{tabular}

Globomycin and congeners
(D)<smiles>CCC(CC)c1nnc(NC(=O)c2cc(OC)c(OC3CCCC3)cc2[N+](=O)[O-])s1</smiles>

Inhibitor-99

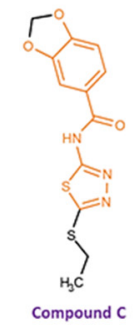

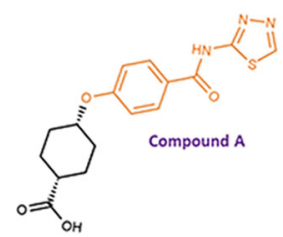

$\mathrm{CH}_{3}$

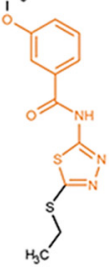

Compound B

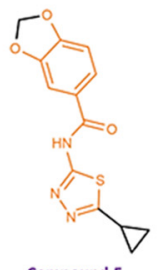

(E)



Myxovirescin A1. C-14 C=C. C-25 $=(R)$



Myxovirescin and congeners

Trends in Biochemical Sciences

Figure 3. The Three Main Inhibitors of LspA and Binding Insights. (A) The structure of LspA (i.e., prolipoprotein signal peptidase, type II signal peptidase, Lsp, SPase II, SPII). The transmembrane helices (TMHs) are numbered. Selected residues (yellow sticks) form direct hydrogen bonds with the cyclic antibiotic globomycin (Figure S4). (A) and (B) were generated using the PyMOL Molecular Graphics System (version 1.7.4.5 Edu, Schrödinger, LLC) and PDB 5DIR [8]. (B) A closer look at bound globomycin. The selected residues in (A) are shown in yellow in the protein ('surface' presentation). Globomycin is in ball-and-stick mode, with the main atoms in magenta, and those generally involved in direct interactions in orange. The main hydroxyl is further labeled as 'dotted sphere' at the catalytic aspartates. (C) Chemical structure of globomycin and analogs SF-1902 A2-A5. Figure adapted, with permission, from [45]. The atoms involved in hydrogen bonding with LspA (based on all chains in PDB 5DIR) are highlighted in blue circles; an orange dashed circle indicates the hydroxyl interacting with the aspartates. (D) Inhibitor-99 (figure adapted, with permission, from [39]) and its isomers identified for this review using Chemicalize (https://chemicalize.com/; developed by ChemAxon, http://www. chemaxon.com). Common features are highlighted in orange. The isomers (named compounds A-F) were described in patents as activators or inhibitors of various proteins (Table S1). (E) The chemical structures of myxovirescin and analogs [50,51] (figure adapted, with permission, from [79]). Myxovirescin B is nearly identical. The labels are hypothetical and are based on the analysis in this figure (B and $\mathrm{C}$ ) and in Figure 4.

be noncompetitive [39], as was previously indicated by an experimental study (inhibitory constant $K_{i}=36 \mathrm{nM}$ ) [40]. We suggest that, when globomycin is tested against the entire enzyme (and in the presence of substrates), the type of inhibition may be identified as 'partially competitive', or noncompetitive vis-à-vis the entire LspA. LspA bound to globomycin may thus still interact with part(s) of the 
prolipoprotein substrate (e.g., the DAG of the cysteine and/or the top part at the exoplasmic leaflet). Globomycin may also bind through a two-step mechanism [38]. The interactions of unbound LspA with the prolipoprotein substrate may involve different parts of the substrate, resulting in more than one product (e.g., cleaved signal peptide and apolipoprotein), and cause multiple conformational changes in LspA (before, during, and after cleavage). Inhibition by globomycin may only correspond to one LspA conformation and involve binding to only a small region of LspA, unlike the interaction of LspA with the entire prolipoprotein substrate (which in particular has membrane and periplasmic parts). This hypothesis highlights the importance of ensuring that, during drug development, interpretation and optimization require a correct understanding both of the enzymology and of the most favorable LspA conformation for designing stronger inhibitors.

Third, the positioning of globomycin within LspA probably occurs through a slow binding process known as steric compression, resulting in a tight hydrogen bond with the aspartates (Figure S4) (e.g., low-barrier hydrogen bonds, LBHBs $[41,42]$ ). Its key hydroxyl sterically blocks the catalytic aspartates Asp124 and Asp143 [8] (Figure 3B). A distorted aspartate coplanarity is likely (e.g., [43]) when globomycin is bound (possibly to an initially dehydrated enzyme) compared to when substrate is bound. There is no place for a water molecule (Figure 3B). Therefore, because the necessary arrangement and geometries may not be present, there is no mimicking of the ideal 'tetrahedral' configuration. This is the case of several other proteins in complex with inhibitors wherein the hydroxyl displaces the water molecule, probably due to slow binding and/or conformational changes during binding. Thus, it is important to verify if globomycin truly mimics the transition state, and to study the LspA conformations.

Fourth, additional stereochemical features are also important in globomycin. Analysis of all chains in the PDB was carried out to identify the main atoms involved in direct hydrogen bonding interactions (Figure 3B,C), including the L-Thr hydroxyl (Figure 3C). Indeed, its stereochemistry was determined to be important for activity $[44,45]$. Some structural similarities with pepstatin (a classic aspartate inhibitor discussed below) are also suggested in Figure S4.

Finally, there are microbiological ambiguities to explore. In Archaea, despite the absence of clear LspA homologs, globomycin specifically inhibits lipoprotein maturation [46]. In mycobacteria, its action may be independent of LspA [47], probably because it inhibits a similar or different enzyme. In some $\mathrm{G}+$ bacteria [18] there are two LspAs (LspA and LspA2), and only LspA is inhibited by globomycin [19].

Inhibitor-99: Recently Discovered but Similar to Known Binders of a Variety of Proteins 'Inhibitor-99', so named here based on its $\mathrm{IC}_{50}$ of 99 nM, was discovered in a high-throughput library screen against LspA $[39,48]$. It was identified (i.e., the tested analogs) as noncompetitive. It is effective in conjunction with the outer-membrane permeabilizer polymyxin B nonapeptide (PMBN). The structure is different from that of either globomycin or myxovirescin (Figure 3D). Our search in chemical databases, including Chemicalize (https://chemicalize.com/; developed by ChemAxon, http://www.chemaxon.com), revealed isomers that were reported to be inhibitors of diverse proteins (Figure 3D and Table S1). Therefore, the specificity (prokaryotic vs eukaryotic proteins) and selectivity should be clinically verified.

Myxovirescin: Different from and More Potent Than Globomycin

Also known as antibiotic TA, megovalicin, and M-230B (Figure 3E), the properties of myxovirescin suggest membrane penetration similarly to globomycin. It belongs to a large macrolactam lactone family and has no toxicity towards eukaryotic cells [49]. Known for half a century, it recently resurfaced as a specific LspA inhibitor that is more potent than globomycin [50,51]. A rarely mentioned derivative known as 'focusin' (hydrogenated form) is more stable but has a lower specific 
activity [52]. A novel assay [39] determined an $\mathrm{IC}_{50}$ of $\sim 1.2 \mathrm{nM}$ for both myxovirescin and globomycin, indicating potency against LspA.

Pepstatin: Research on Its Derivatives Is Valuable for Enhancing Existing LspA Inhibitors

Because LspA is an aspartate protease, the peptide pepstatin - a classic inhibitor of several aspartate proteases - is reviewed here. There are few studies on its effects on LspA [53], but one paper reported an $\mathrm{IC}_{50}$ of $0.32 \mathrm{mM}$ [54]. Generally, pepstatin interacts with targets through a hydrogen bond network (Figure 4). The $y$-amino acid statine within pepstatin makes pepstatin nonhydrolyzable and a tetrahedral transition state mimic, and is also incorporated into many HIV protease and renin inhibitors [55]. Nevertheless, as with globomycin, the main hydroxyl at the aspartates may in some cases be tightly bound without space for water (see above). Designs with statine-based cores are common (Figure 4), for example 'inhibitor a' [5] against plasmepsins [56] and a phosphonated

(A)

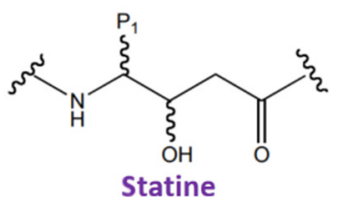<smiles>CCNC(=P)[C@H](O)CNC</smiles>

Hydroxyethylamine<smiles>[R7]C(=O)N[C@@H](P)[C@H](O)CN([PH])C(=O)[PH]</smiles>

Hydroxyethylamine core

(B)<smiles>[R7]NC(=O)C[C@H](O)[C@H](P)NC([R7])=O</smiles>

Statine-derived core<smiles>[R7]C(=O)NC[C@@H](O)[C@H](O[PH3+])C(=O)N[PH3]</smiles>

Reversed-statine-based isosteres $(R, R)$ and $(S, S)$
(C)

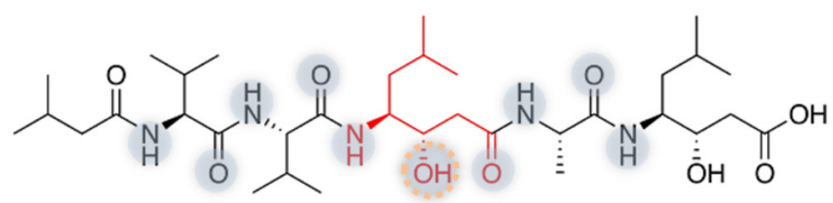

Pepstatin A

(Iva-Val-Val-Sta-Ala-Sta)

(D)



Phosphonate inhibitor

(Iva-Val-Val-LeuP-(O)Phe-Ala-Ala-OMe)<smiles>CC(=O)NN(Cc1ccccc1)C[C@H](O)[C@@H](OCc1ccccc1)C(=O)NCCN1CCCCC1</smiles>

Aspartyl protease inhibitor a<smiles>O=C(NP)[C@@H]([OH2+])[C@H](O)CNC(=P)P</smiles> 
form that forms a short hydrogen bond [57]. The hydroxyl may be also replaced by other atoms. We believe that there is structural similarity with globomycin (Figure S4). If derivatives against LspA are developed, their cell penetration ability [58] should be ensured. Perhaps lessons can be drawn from developments for other intramembrane proteases (e.g., rhomboids [59]).

\section{Lnt: A Nitrilase with Many Unique Features}

A Catalytic Tetrad C-E-E-K in the Nitrilase Domain Is Close to the Transmembrane (TM) Domain Lnt is an unusual nitrilase (reverse amidase [60]) that is connected to a TM domain [9-12] (Figure 5). The nitrilase superfamily has a conserved Glu-Lys-Cys catalytic triad (prokaryotes and eukaryotes) [60]. However, some nitrilases have a tetrad (C-E-E-K) owing to a fourth conserved catalytic residue (Glu) (e.g., formamidases [61]). In Lnt (triad E267-K335-C387), the conserved E343 near the triad is almost fixed in all structures [62], despite its proximity to highly flexible regions (Figure 5A). E343 may facilitate recognition of the donor lipid as well as the formation of the Lnt-lipid intermediate and stabilization of K335 in the triad [10], thus it is important for catalysis overall, particularly for the lipid-binding reaction (first step). Likewise, in some amidases, a similar Glu residue was proposed to be involved in substrate positioning [63]. Thus, as in nitrilases previously [61], we suggest also naming the Lnt catalytic element a 'tetrad'. Other unique features in Lnt are a lidloop (that may protect the binding cavity) and a phosphate-binding site.

A Long Flexible Loop May Act as a Lid to Restrict Access of Molecules from the Periplasm A unique loop (compared to soluble nitrilases) contains a short helix and a flexible amphipathic lidloop (F357-Q372) [10] that may enforce its membrane anchoring. As determined from six Lnt structures, the sequence of highest divergence and flexibility is F344-S363 (Figure 5A) [62]. Therefore, here, based on the comparisons of this entire sequence, we describe the lid-loop and its conformations differently, and may call it 'loop 3xx'. Its electron density is partly missing in most structures. Clearly, it is adjacent to E343 and may identify a potential druggable site (Figure S1).

The loop likely restricts periplasmic access to the binding groove of Lnt by free molecules not originating from the membrane (Figure 5A, left) by controlling the interface as a lid that maintains contact with the membrane. Therefore, only lipid donors from the membrane are able to bind. Hypothetically, when the loop is raised (high position), it acts as a closed lid, and when lowered and to the side, following the lipid-donor binding, it adopts the 'open' position (Figure 5A, middle). Therefore, it is also expected that the open/lowered position permits the anchorage of the apolipoprotein (Figure 1) at the cavity of Lnt. As a side note, residue F82 (opposite to the loop) that was previously proposed to be a gatekeeper [9] (for the lipid donor at the binding site, assuming that the loop is in the low position at all times) appears not to play such a role in the present lid-loop (low vs high) hypothesis, and this would also explain why a F82 mutant [9] had no effect.

During the first step of the reaction, part of the lipid donor forms the Lnt-acyl intermediate, while the other part is released. The acceptor apolipoprotein then becomes anchored for the second step of the reaction. Note that some structures were solved with the loop in the 'open' position, and do not contain relevant bound lipids [11]. Which loop conformation was obtained in a structure probably depends on the crystallization method used and crystal packing. A very recent study also suggested this possibility [12], to be due for example to the added detergents, the presence of artificial lipids during crystallization, and/or crystalline molecular packing. This study identified a Lnt intermediate form (thioester acyl intermediate, chain B; PDBs not available), with an even more upward (higher) loop compared to that labeled in the red ribbon in Figure 5A. It is unlikely that this new conformation permits the loop stabilization with the membrane, or the binding of the apolipoprotein, also because in several PDBs with bound lipids the loop was in the open/low position (yellow ribbon in Figure 5A). Therefore, the positions proposed in Figure 5A may be more likely. Analysis of the loop is important to consider when designing inhibitors, in 
(A)
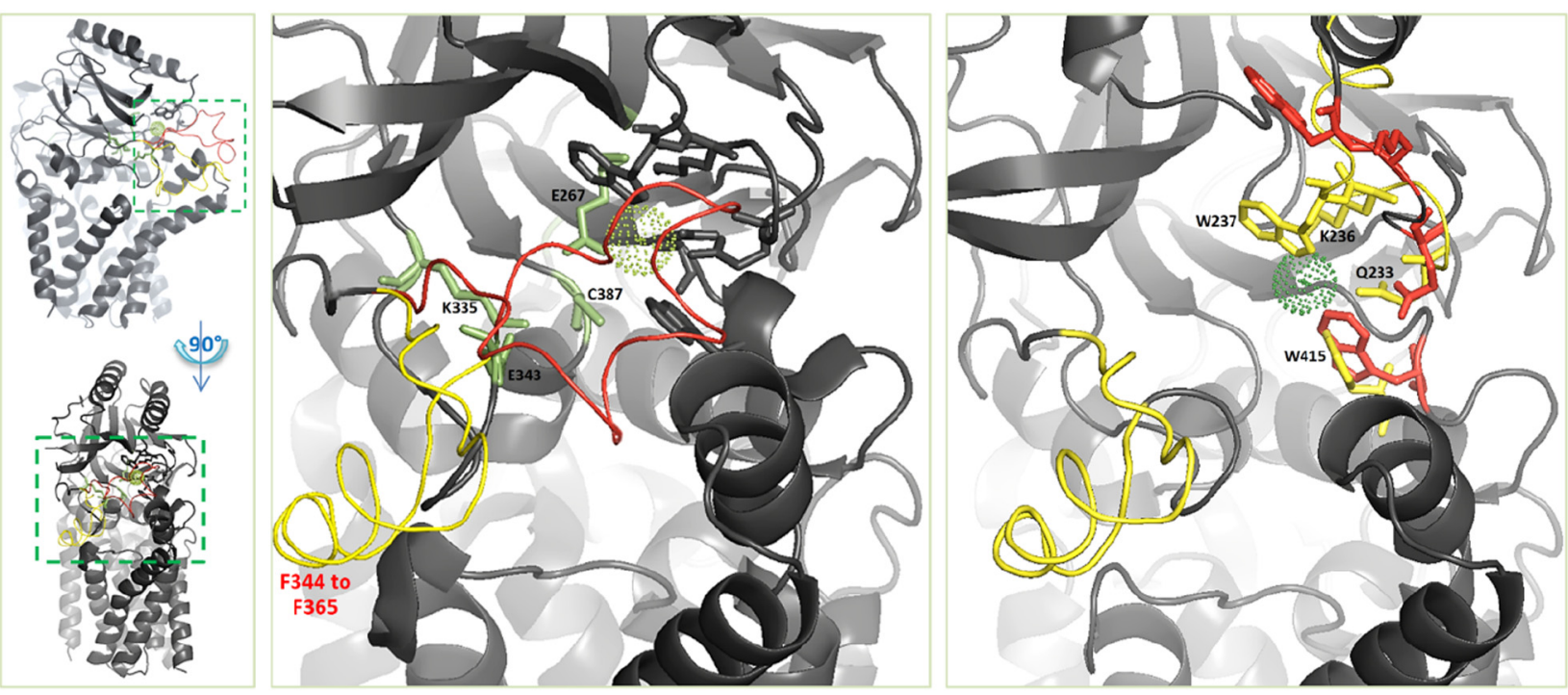

(B)

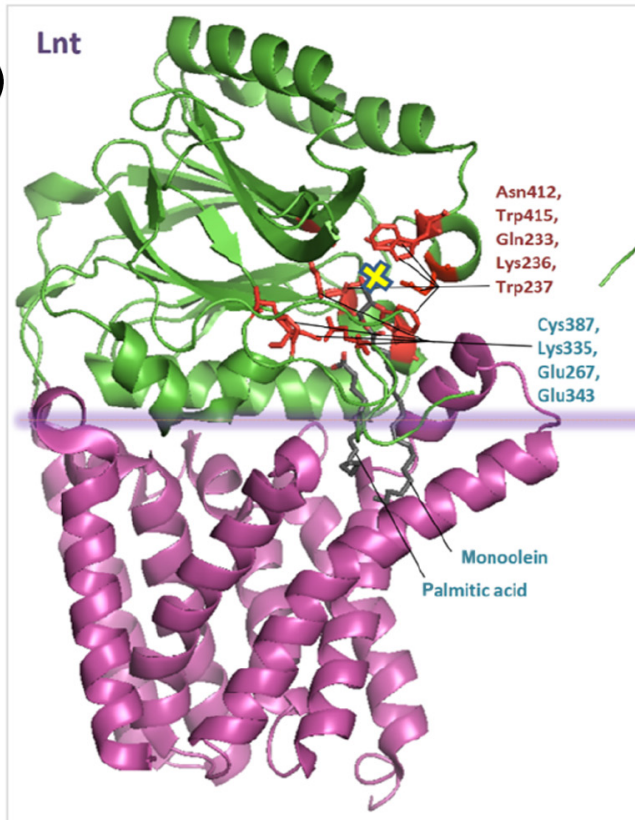

$\mathrm{PlsC}$

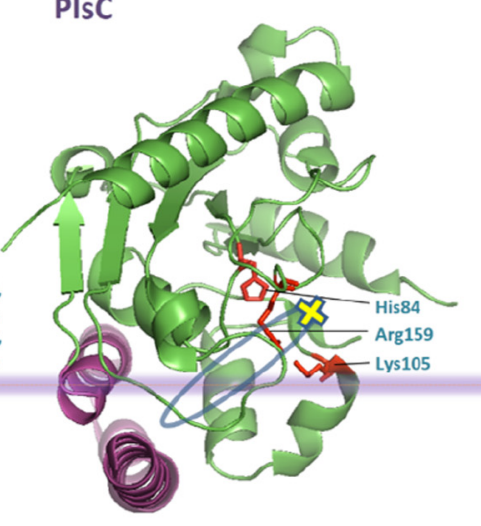

DHHC20

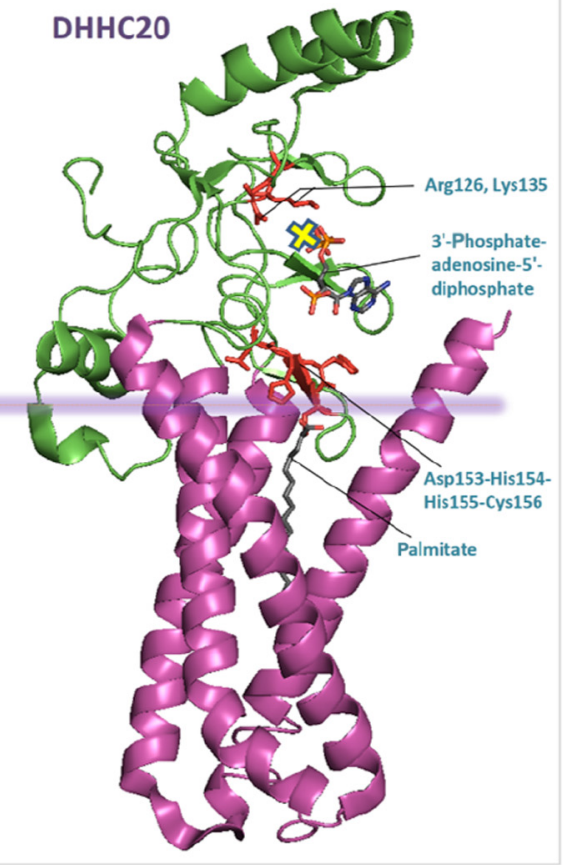

Trends in Biochemical Sciences

Figure 5. Conformation Models of the Lid-Loop and the Phosphate-Binding Site of Lnt. (A) Flexibilities of the loop and the phosphate-binding residues. (Left) Lnt structure (PDB 5XHQ [10] was used as a template). The two conformations of the proposed lid-loop ('loop 3xx'), closed/raised and open/lowered, are as observed in PDB $5 \mathrm{XHQ}$ (red ribbon, invisible density manually completed here) and PDB 5N6H [11] (yellow ribbon), respectively. When raised, it restricts against periplasmic molecules but allows lipid donor access from the membrane leaflet. Then, in the open position in the Lnt-lipid intermediate, the apolipoprotein may bind. (Middle) A closer view, with the catalytic tetrad shown as green sticks (E267-K335-E343-C387). The green dotted sphere represents the chloride superimposed from PDB 5VRH [9]. (Right) Potential phosphate-binding residues. Residues W237, K236, Q233, and W415 are highlighted as red (PDB 5N6H [11]) or yellow sticks (PDB 5VRH [9]) (loop in open position in both PDB structures). The difference may correspond to stabilization by, and interaction, with an element such as chloride (green sphere) or the head group of the lipid donor. (B) Three acyltransferases, Lnt, PISC, and a DHHC enzyme, that rely on the membrane. The purple horizontal line is an approximation of the level of the membrane, and the protein features in magenta represent the transmembrane helices in Lnt (PDB 5VRG) and DHHC2O (Golgi body membrane, PDB 6BMN) or the two-helix anchoring motif in PISC (to the inner leaflet of the inner membrane) (PDB 5KYM) [9,69,70]. The yellow cross indicates the potential binding locations of the phosphate groups of ligands. Bound acyl chains in Lnt and DHHC20 can be seen, while in PlsC (acyl donor not shown) the drawn oval indicates the potential position of lysophosphatidic acid [70]. Selected residues are highlighted as red sticks. The figures were generated using the PyMOL Molecular Graphics System (version 1.7.4.5 Edu, Schrödinger, LLC). 
assessing whether they will bind, and in determining whether other designs should be explored to block the Lnt-lipid intermediate in the open loop mode.

\section{A Pocket for Stabilizing the Lipid Head Group Shows Flexibility}

The phosphate recognition site in Lnt was hypothesized [9] based on a chloride ion interacting with several residues (Q233, W237, K236, N412, W415) above the catalytic tetrad. These interaction residues include important and characterized residues [9,64] (Figure 5A). Interestingly, soluble nitrilases may not contain such a pocket owing to further sequence extensions (Figure S5). Comparison of all Lnt structures revealed flexibilities mainly of four of the phosphate interaction residues, as shown in Figure 5A, but N412 was not highlighted because it has a similar position in all PDB structures. Furthermore, based on the lid-loop hypothesis, this site may also be protected against periplasmic molecules, and may mediate the selectivity and stabilization of the lipid donor (e.g., PE, PG, or phosphatidic acid [65]) by binding its head group (e.g., POPE) [9]). In the next reaction step, namely the binding and interaction with the protein acceptor, it is also possible that the phosphate recognition site facilitates the docking of the apolipoprotein, and perhaps interacts with residues $+2 /+3 /+4$ that follow the $\mathrm{N}$-terminal conserved Cys ${ }^{+1}$ (Figure 1). The $+2 /+3 /+4$ residues have been shown to be important for the interaction of the lipoprotein with complexes in other pathways [66-68] (see Outstanding Questions). Unfortunately, it is unclear why the phosphate interaction residues are in the same position in both $\mathrm{PDBs} 5 \mathrm{XHQ}$ (loop as 'closed lid') and 5VRH (open loop) (Figure 5A), despite a $\mathrm{H}_{2} \mathrm{O}$ built in the place of the chloride, and it is also unclear to what extent the position of the loop has an effect on these residues.

The recently solved structures of membrane-dependent acyltransferases from other families, PIsC [1-acyl-sn-glycerol-3-phosphate (LPA) acyltransferase (LPAAT)] and DHHC2O [palmitoyltransferase (S-acyltransferase)] [69,70], also suggest the presence of a phosphatebinding site (Figure 5B). The bacterial PIsC is anchored to the inner membrane (facing the cytoplasm) and uses lysoPA as a substrate that is usually generated by PIsB (G3P acyltransferase, GPAT), to form phosphatidic acid (PA). PlsC contains a catalytic His84, a transition-state stabilizer Lys 105, and Arg159 which may (mainly) bind the 3'-phosphate of lysoPA [70] (Figure 5B). The eukaryotic palmitoyltransferase DHHC20 [71] (human S-acyltransferase) is a Golgi TM protein with a cytoplasmic domain that uses acyl-CoA donors (as do PlsB and PlsC) such as palmitoyl-CoA, and has a catalytic DHHC motif. In Figure 5B, the physiological orientation of DHHC20 (Golgi body membrane) is towards the cytoplasm, as is PlsC (cytoplasmic membrane), whereas Lnt (cytoplasmic membrane) is towards the periplasm. Furthermore, several DHHC2O residues, of which two are labeled at the top (Figure 5B), interact with phosphate groups [69].

Inhibitor Design Against Lnt: What Can Soluble Nitrilases Tell Us?

As previously mentioned, Lnt has both a nitrilase and TM domains (Figures 1 and 5). For soluble nitrilases, an acyl-transfer intermediate (acetohydroxamic acid derivative) was identified in an amidase structure [72], which is interesting because it is covalently bound. Furthermore, antiparasitic lipophilic compounds, derivatives of acetohydroxamic acid, were tested [73,74]. Such studies may guide the design of inhibitors of Lnt acyltransferase, and this will likely require hydrophobic inhibitors that access from the membrane on which Lnt depends, according to its lid-loop restriction against free periplasmic molecules. In Lnt, lipid acyl chains also extend almost vertically below the catalytic residues (Figure 5), as do ligands in soluble nitrilases (Figure S6). In addition, relevant inhibitor enhancement functionalities may for example be halogens (for $\mathbf{X}$-bonding), as previously reported for a nitrilase [75]. Halogen bonds can be specific, directional, and noncovalent, with a positive impact on binding and selectivity [76]. Furthermore, allosteric nitrilase or membrane domain sites may be also possible (Figure S1), but are probably less specific. Perhaps the acyl-Lnt intermediate with an open loop as in Figure 5A, or the non-bound phosphate site (for a small 
inhibitor probably accessing near the lid-loop), may be also possible to target with other inhibitor candidates; however, in this case it will be difficult to learn much from soluble nitrilases.

\section{Concluding Remarks and Future Perspectives}

Bacterial species possess several different modification enzymes and lipoprotein forms. In particular, Lgt, LspA, and Lnt were investigated in view of their central roles in lipoprotein biogenesis, and to establish priorities for drug design pipelines. Lgt likely involves a two-step process with an induced-fit conformation. It binds common lipids, and has structural similarities with other proteins such as PgpB and PIsY, which raises the challenge of developing specific inhibitors. However, the other cleft for binding to the pre-prolipoprotein lipobox motif might be more suitable for targeting, particularly if there is no need to inhibit the lipid-binding cleft simultaneously and if the inhibitor binds to the correct Lgt conformation that mostly prohibits binding of the preprolipoprotein. Note that in some species an additional Lgt may be present [20], and LspA may act independently of Lgt $[18,23]$. As for LspA, it has the simplest structure, and has no homologs in eukaryotes. Four molecules have been discussed in the context of inhibitor design against LspA, and provide interesting clues compared to current data on Lgt and Lnt. Lnt seems, compared to Lgt or LspA, less essential in many species or even not present [14,15,20,21,24,25]. Furthermore, several enzymes (e.g., Lit or unusual Lnts) other than the E. coli-type Lnt may carry out modifications $[20,21,24,25]$. Pursuit of Lnt inhibitors will require that the specific dynamics of the structure (e.g., the loop and phosphate-binding site) are further characterized, and may provide advantages compared to soluble nitrilases [60].

Overall, LspA is recommended for future drug discovery pipelines over Lgt and Lnt. Note, however, that there will be limitations to the range of pathogens that can be targeted based on their cellular barriers and the presence of a non-essential LspA or multiple LspAs [16-19,37]. Potential antibiotics should be also tested in different species to determine whether they might act similarly to globomycin that can be LspA-independent $[46,47]$. One potential advantage of targeting LspA is that the solved variant structure of LspA [8] was based on the Pseudomonas aeruginosa protein (one of the ESKAPE pathogens). Furthermore, the knowledge of several inhibitors presented here may be combined with that of known engineered pepstatins, and implemented into novel designs, while taking into consideration the described hypotheses on the mechanism of LspA inhibition based on globomycin.

In terms of hypothetical drug design, organic chemistry seems to be crucial to enhance the stability and efficacy of lipophilic peptidomimetics [77,78], as well as their permeability across the lipopolysaccharide layer of $\mathrm{G}$ - bacteria $[36,79]$, while minimizing clinical challenges (ADMET). An accelerated route might be to explore microorganisms for natural products [80], as in the case of globomycin [34,35] and myxovirescins [51] or other macrolides (with a lactone ring [81]), and to study bound structures and conformations using powerful structural biology techniques $[82,83]$ (see Outstanding Questions).

\section{Acknowledgments}

The authors would like to thank Michael P. Ryan, Michele Dully, KimLeah Shortall, the Editor, and the editorial board of the journal for valuable comments.

\section{Supplemental Information}

Supplemental information associated with this article can be found online at https://doi.org/10.1016/j.tibs.2019.03.007.

\section{Outstanding Questions}

Given the varying extents of essentiality, homology, and significance of Lgt, LspA, and Lnt across different bacterial species, how effective will a developed drug be against target species and in bypassing different resistance mechanisms?

Will a promising (in vitro) lipophilic antibiotic pass clinical trials and cause neither high toxicity nor random membrane disruption?

What are the structures of the functiona homologs of Lgt, LspA, and Lnt, as well as of deacylase and Lit?

Lgt: given two binding clefts, ordinary lipid binding, and an induced-fit structure, should (i) the pre-prolipoproteinbinding cleft or (ii) an allosteric site be explored for drug design instead of the lipid-binding cleft?

LspA: regarding globomycin, (i) is it a partially noncompetitive inhibitor based on the hypotheses presented, (ii) does it inhibit by mimicking the binding of the signal peptide linked to the prolipoprotein or the signal peptide product after cleavage (i.e., residues $-3,-2,-1$ ), and (iii) what does it bind to in Archaea and mycobacteria?

LspA: how potent will globomycin myxovirescin, and inhibitor-99 analogs be following engineering and the addition of core functionalities according to pepstatin derivatives?

Lnt: at/during the second reaction step, are there specific interactions between its proposed phosphate-binding site and the $\mathrm{N}$-terminal Lol sorting signal of the apolipoprotein (and perhaps the tether region)?

Lnt: (i) is there a relationship between the phosphate-binding site and the lid-loop conformation? (ii) What is the exact movement of the loop as a protective lid, its interaction with donor lipids, and its conformation throughout the entire reaction? Alternatively, (iii) is the loop in the 'low' (open) mode at all times?

\section{References}

1. Johnson, T.W. et al. (2009) Using the golden triangle to optimize clearance and oral absorption. Bioorg. Med. Chem. Lett. 19, $5560-5564$
2. Lipinski, C.A. et al. (2001) Experimental and computational approaches to estimate solubility and permeability in drug discovery and development settings. Adv. Drug Deliv. Rev. 46, 3-26 
3. Atangcho, L. et al. (2019) Hitting undruggable targets: viewing stabilized peptide development through the lens of quantitative systems pharmacology. Trends Biochem. Sci. 44, 241-257

4. Ettari, R. et al. (2010) Falcipain-2 inhibitors. Med. Res. Rev. 30, 136-167

5. Perez, B. et al. (2013) Development of Plasmodium falciparum protease inhibitors in the past decade (2002-2012). Curr. Med. Chem. 20, 3049-3068

6. Mallinson, J. and Collins, I. (2012) Macrocycles in new drug discovery. Future Med. Chem. 4, 1409-1438

7. Mao, G. et al. (2016) Crystal structure of E. coli lipoprotein diacylglyceryl transferase. Nat. Commun. 7, 10198

8. Vogeley, L. et al. (2016) Structural basis of lipoprotein signa peptidase II action and inhibition by the antibiotic globomycin Science 351, 876-880

9. Noland, C.L. et al. (2017) Structural insights into lipoprotein N acylation by Escherichia coli apolipoprotein $\mathrm{N}$-acyltransferase. Proc. Natl. Acad. Sci. U. S. A. 114, E6044-E6053

10. Lu, G. et al. (2017) Crystal structure of $E$. coli apolipoprotein Nacyl transferase. Nat. Commun. 8, 15948

11. Wiktor, M. et al. (2017) Structural insights into the mechanism of the membrane integral $\mathrm{N}$-acyltransferase step in bacterial lipoprotein synthesis. Nat. Commun. 8, 15952

12. Wiseman, B. and Hogbom, M. (2018) Conformational changes in apolipoprotein $\mathrm{N}$-acyltransferase (Lnt). bioRxiv. Published online December 16, 2018. https://doi.org/10.1101/497412

13. Sankaran, K. and Wu, H.C. (1994) Lipid modification of bacteria prolipoprotein. Transfer of diacylglyceryl moiety from phosphatidylglycerol. J. Biol. Chem. 269, 19701-19706

14. LoVullo, E.D. et al. (2015) Revisiting the Gram-negative lipopro tein paradigm. J. Bacteriol. 197, 1705-1715

15. Gwin, C.M. et al. (2018) The apolipoprotein N-acyl transferase Lnt is dispensable for growth in Acinetobacter species. Microbiology 164, 1547-1556

16. Konovalova, A. et al. (2010) Extracellular biology of Myxococcus xanthus. FEMS Microbiol. Rev. 34, 89-106

17. Becker, K. and Sander, P. (2016) Mycobacterium tuberculosis lipoproteins in virulence and immunity - fighting with a doubleedged sword. FEBS Lett. 590, 3800-3819

18. Wooldridge, K. (2009) Bacterial Secreted Proteins: Secretory Mechanisms and Role in Pathogenesis, Caister Academic Press

19. Charlton, Thomas M. et al. (2015) Quantitative lipoproteomics in Clostridium difficile reveals a role for lipoproteins in sporulation. Chem. Biol. 22, 1562-1573

20. Widdick, D.A. et al. (2011) Dissecting the complete lipoprotein biogenesis pathway in Streptomyces scabies. Mol. Microbiol. 80, 1395-1412

21. Kurokawa, K. et al. (2009) The triacylated ATP binding cluster transporter substrate-binding lipoprotein of Staphylococcus aureus functions as a native ligand for Toll-like receptor 2. J. Biol. Chem. 284, 8406-8411

22. Storf, S. et al. (2010) Mutational and bioinformatic analysis of haloarchaeal lipobox-containing proteins. Archaea 2010 410975

23. Baumgartner, M. et al. (2007) Inactivation of Lgt allows systematic characterization of lipoproteins from Listeria monocytogenes. J. Bacteriol. 189, 313-324

24. Armbruster, K.M. and Meredith, T.C. (2017) Identification of the lyso-form $\mathrm{N}$-acyl intramolecular transferase in low-GC Firmicutes. J. Bacteriol. 199, e00099-17

25. Kurokawa, K. et al. (2012) Novel bacterial lipoprotein structures conserved in low-GC content Gram-positive bacteria are recognized by Toll-like receptor 2. J. Biol. Chem. 287 , 13170-13181

26. Cho, H. et al. (2011) Molecular mechanism by which palmitate inhibits PKR autophosphorylation. Biochemistry 50 1110-1119

27. Fujishiro, T. et al. (2011) Crystal structure of $\mathrm{H}_{2} \mathrm{O}_{2}$-dependent cytochrome P450SPalpha with its bound fatty acid substrate: insight into the regioselective hydroxylation of fatty acids at the alpha position. J. Biol. Chem. 286, 29941-29950

28. Zhu, G. et al. (2013) Crystal structures of alpha-dioxygenase from Oryza sativa: insights into substrate binding and activation by hydrogen peroxide. Protein Sci. 22, 1432-1438
29. Tang, Y. et al. (2018) Membrane phospholipid biosynthesis in bacteria, In Advances in Membrane Proteins. Part I: Mass Processing and Transportation (Cao, Y., ed), pp. 77-119, Springer, Singapore

30. Fan, J. et al. (2014) Crystal structure of lipid phosphatase Escherichia coli phosphatidylglycerophosphate phosphatase B. Proc. Natl. Acad. Sci. U. S. A. 111, 7636-7640

31. Ovchinnikov, S. et al. (2015) Large-scale determination of previously unsolved protein structures using evolutionary information. eLife 4, e09248

32. Tong, S. et al. (2016) Structural insight into substrate selection and catalysis of lipid phosphate phosphatase PgpB in the cell membrane. J. Biol. Chem. 291, 18342-18352

33. Li, Z. et al. (2017) Structural insights into the committed step of bacterial phospholipid biosynthesis. Nat. Commun. 8, 1691

34. Inukai, M. et al. (1978) Globomycin, a new peptide antibiotic with spheroplast-forming activity. I. Taxonomy of producing organisms and fermentation. J Antibiot. (Tokyo) 31, 410-420

35. Omoto, S. et al. (1979) Isolation and structure of SF-1902 A5, a new globomycin analogue. J Antibiot. (Tokyo) 32, 83-86

36. Lai, J.S. et al. (1981) Globomycin sensitivity of Escherichia coll and Salmonella typhimurium: effects of mutations affecting structures of murein lipoprotein. J. Bacteriol. 145, 657-660

37. Omoto, S. et al. (1981) Studies on SF-1902 A2 A5, minor components of SF-1902 (globomycin). J. Antibiot. 34, 1416-1423

38. Blat, Y. (2010) Non-competitive inhibition by active site binders. Chem. Biol. Drug Des. 75, 535-540

39. Kitamura, S. et al. (2018) Lipoprotein signal peptidase inhibitors with antibiotic properties identified through design of a robust in vitro HT platform. Cell Chem. Biol. 25, 301-308

40. Dev, I.K. et al. (1985) Inhibition of prolipoprotein signal peptidase by globomycin. J. Biol. Chem. 260, 5891-5894

41. Erskine, P.T. et al. (2003) Atomic resolution analysis of the cataIytic site of an aspartic proteinase and an unexpected mode of binding by short peptides. Protein Sci. 12, 1741-1749

42. Cleland, W.W. et al. (1998) The low barrier hydrogen bond in enzymatic catalysis. J. Biol. Chem. 273, 25529-25532

43. Toulokhonova, L. et al. (2003) Kinetic studies on $\beta$-site amyloid precursor protein-cleaving enzyme (BACE): confirmation of an iso-mechanism. J. Biol. Chem. 278, 4582-4589

44. Kiho, T. et al. (2003) Synthesis and antimicrobial activity of nove globomycin analogues. Bioorg. Med. Chem. Lett. 13, 2315-2318

45. Kiho, T et al (2004) Structure-activity relationships of globomycin analogues as antibiotics. Bioorg. Med. Chem. 12, 337-361

46. Giménez, M.I. et al. (2007) Haloferax volcanii twin-arginine translocation substates include secreted soluble, C-terminally anchored and lipoproteins. Mol. Microbiol. 66, 1597-1606

47. Banaiee, N. et al. (2007) LspA-independent action of globomycin on Mycobacterium tuberculosis. J. Antimicrob. Chemother. 60, $414-416$

48. Wolan, D.W. and Owensby, A. Scripps Research Institute. Methods for identifying novel antibiotics and related compositions, WO2017040762A2

49. Rosenberg, E. and Dworkin, M. (1996) Autocides and a paracide, antibiotic TA, produced by Myxococcus xanthus. J. Ind. Microbiol. 17, 424-431

50. Xiao, Y. et al. (2012) Myxobacterium-produced antibiotic TA (myxovirescin) inhibits type II signal peptidase. Antimicrob. Agents Chemother. 56, 2014-2021

51. Rosenberg, E. et al. (1973) Bactericidal action of an antibiotic produced by Myxococcus xanthus. Antimicrob. Agents Chemother. 4, 507-513

52. Rosenberg, E. Ramot University Authority. Antibacterial agent, US5532231A

53. El Arnaout, T. (2013) Structure-Function Studies of the Prolipoprotein Signal Peptidase, LspA, Trinity College

54. Dev, I.K. and Ray, P.H. (1984) Rapid assay and purification of unique signal peptidase that processes the prolipoprotein from Escherichia coli B. J. Biol. Chem. 259, 11114-11120

55. Gacko, M. et al. (2007) Cathepsin D inhibitors. Folia Histochem Cytobiol. 45, 291-313

56. Dahlgren, A. et al. (2003) New inhibitors of the malaria asparty proteases plasmepsin I and II. Bioorg. Med. Chem. 11, 3423-3437 
57. Masao, F. et al. (2000) Structural study of the complex between human pepsin and a phosphorus-containing peptidictransitionstate analog. Acta Crystallogr. Sect. D 56, 272-279

58. Takagi, Y. et al. (2017) Discovery of novel cyclic peptide inhibitors of dengue virus NS2B-NS3 protease with antiviral activity. Bioorg. Med. Chem. Lett. 27, 3586-3590

59. Tichá, A. et al. (2018) The rhomboid superfamily: structural mechanisms and chemical biology opportunities. Trends Biochem. Sci. 43, 726-739

60. Pace, H.C. and Brenner, C. (2001) The nitrilase superfamily: classification, structure and function. Genome Biol. 2, REVIEWS0001

61. Soriano-Maldonado, P. et al. (2011) Biochemical and mutational studies of the Bacillus cereus CECT 5050T formamidase support the existence of a C-E-E-K tetrad in several members of the nitrilase superfamily. Appl. Environ. Microbiol. 77, 5761-5769

62. Cheng, W. et al. (2018) The N-acyltransferase Lnt: structurefunction insights from recent simultaneous studies. Int. J. Biol. Macromol. 117, 870-877

63. Weber, B.W. et al. (2013) The mechanism of the amidases: mutating the glutamate adjacent to the catalytic triad inactivates the enzyme due to substrate mispositioning. J. Biol. Chem. 288, 28514-28523

64. Gelis-Jeanvoine, S. et al. (2015) Residues located on membrane-embedded flexible loops are essential for the second step of the apolipoprotein $\mathrm{N}$-acyltransferase reaction. Mol. Microbiol. 95, 692-705

65. Hillmann, F. et al. (2011) Kinetics and phospholipid specificity of apolipoprotein N-acyttransferase. J. Biol. Chem. 286, 27936-27946

66. Narita, S. and Tokuda, H. (2007) Amino acids at positions 3 and 4 determine the membrane specificity of Pseudomonas aeruginosa lipoproteins. J. Biol. Chem. 282, 13372-13378

67. Anke, S. et al. (1999) Testing the '+2 rule' for lipoprotein sorting in the Escherichia coli cell envelope with a new genetic selection. Mol. Microbiol. 34, 810-821

68. Zuckert, W.R. (2014) Secretion of bacterial lipoproteins: through the cytoplasmic membrane, the periplasm and beyond. Biochim. Biophys. Acta 1843, 1509-1516

69. Rana, M.S. et al. (2018) Fatty acyl recognition and transfer by an integral membrane S-acyltransferase. Science 359, eaao6326

70. Robertson, R.M. et al. (2017) A two-helix motif positions the lysophosphatidic acid acyltransferase active site for catalysis within the membrane bilayer. Nat. Struct. Mol. Biol. 24, 666-671

71. Lanyon-Hogg, T. et al. (2017) Dynamic protein acylation: new substrates, mechanisms, and drug targets. Trends Biochem. Sci. $42,566-58$
72. Andrade, J. et al. (2007) Structure of amidase from Pseudomonas aeruginosa showing a trapped acyl transfer reaction intermediate state. J. Biol. Chem. 282, 19598-19605

73. Fytas, C. et al. (2011) Novel lipophilic acetohydroxamic acid derivatives based on conformationally constrained spiro carbocyclic 2,6-diketopiperazine scaffolds with potent trypanocidal activity. J. Med. Chem. 54, 5250-5254

74. Zoidis, G. et al. (2018) Lipophilic conformationally constrained spiro carbocyclic 2,6-diketopiperazine-1-acetohydroxamic acid analogues as trypanocidal and leishmanicidal agents: an extended SAR study. Chem. Biol. Drug Des. 91, 408-421

75. Jiang, S. et al. (2016) The important role of halogen bond in substrate selectivity of enzymatic catalysis. Sci. Rep. 6, 34750

76. Mendez, L. et al. (2017) Looking back, looking forward at halogen bonding in drug discovery. Molecules 22, E1397

77. Delcour, A.H. (2009) Outer membrane permeability and antibiotic resistance. Biochim. Biophys. Acta 1794, 808-816

78. Ghai, I. and Ghai, S. (2017) Exploring bacterial outer membrane barrier to combat bad bugs. Infect. Drug Resist. 10, 261-273

79. Brown, D.G. et al. (2014) New natural products as new leads for antibacterial drug discovery. Bioorg. Med. Chem. Lett. 24 413-418

80. Hertweck, C. (2015) Decoding and reprogramming complex polyketide assembly lines: prospects for synthetic biology. Trends Biochem. Sci. 40, 189-199

81. Vázquez-Laslop, N. and Mankin, A.S. (2018) How macrolide antibiotics work. Trends Biochem. Sci. 43, 668-684

82. Johansson, L.C. et al. (2017) A bright future for serial femtosec ond crystallography with XFELs. Trends Biochem. Sci. 42, 749-762

83. Bai, X.-C. et al. (2015) How cryo-EM is revolutionizing structural biology. Trends Biochem. Sci. 40, 49-57

84. Krishnamoorthy, G. et al. (2017) Synergy between active efflux and outer membrane diffusion defines rules of antibiotic permeation into Gram-negative bacteria. mBio 8, e01172-17

85. Pletzer, D. et al. (2018) Synergy between conventional antibiotics and anti-biofilm peptides in a murine, sub-cutaneous abscess model caused by recalcitrant ESKAPE pathogens. PLOS Pathog. 14, e1007084

86. Deloitte Centre for Health Solutions (2018) Embracing the Future of Work to Unlock R\&D Productivity - Measuring the Return from Pharmaceutical Innovation, Deloitte LLP

87. Fujinaga, M. et al. (1995) Crystal structure of human pepsin and its complex with pepstatin. Protein Sci. 4, 960-972

88. Rose, A.S. et al. (2018) NGL viewer: web-based molecular graphics for large complexes. Bioinformatics 34, 3755-3758 